\title{
Genre et psychanalyse, des outils pour les études cinématographiques
}

Genre et psychanalyse, la différence des sexes en questions, éd. Erés, 2016

Caroline Renard

Aix Marseille Univ, LESA, Aix-en-Provence, France

Du point de vue des études cinématographiques, nous devons distinguer ce que le cinéma nous apprend de la psychanalyse et des questions de genre, de ce que ces champs de recherche nous apprennent, en retour, du cinéma. Cinéma et psychanalyse sont des disciplines historiquement jumelles. Le mot psychanalyse apparaît dans un texte de Freud un an après la première projection d'une vue Lumière à Paris ${ }^{1}$ et les études filmiques se sont emparées des concepts de Freud et de Lacan dès les années $1970^{2}$. Cinéma et psychanalyse ont donc « des choses à se dire $»^{3}$. Les études filmiques françaises sont, en revanche, encore réservées dans leur usage de la notion de genre. Il faut rappeler que dans la langue française ce mot reste plus polysémique et flou que son modèle anglophone gender. Il possède une longue histoire en linguistique, en biologie ou en arts, et il a profité de sa plasticité sémantique pour s'ouvrir aux sciences sociales. Le concept de genre sert à y désigner les différences non biologiques entre les êtres et tend à se confondre avec la notion de « rapports sociaux de sexe ». Ce concept renvoie à la dimension conflictuelle et inégalitaire entre hommes et femmes et dessine les catégories et les formes de pouvoir. Les problématiques qui lui sont liées traversent de fait différentes disciplines. Les études de genre ont fédéré une bonne partie des études féministes ; elles ont focalisé leur attention sur la construction des identités et des significations sociales et observent comment les productions culturelles participent des « technologies » de pouvoir sur les corps. La notion de genre demeure cependant peu convoquée dans les réflexions sur l'esthétique ou les théories du cinéma.

Articuler «genre », "psychanalyse » et « cinéma » n'est pas aisé. La question des identités qui animent les études de genre ne serait pas celle de la psychanalyse mais plutôt celle de la sociologie

1 "Nouvelles remarques sur les psychonévroses de défense", Névrose, psychose et perversion, 1896, PUF, reéd 2010, p.61-83

2 Voir notamment les travaux de Raymond Bellour et Laura Mulvey

3 C.Clément "Un long flirt, une longue acoquinade", CinémAction n50, Cinéma et Psychanalyse, 1989 , p.12 
ou de l'histoire. En revanche la question du sujet au sens psychanalytique intéresse les études du genre. En cinéma travailler sur les rapports sociaux de sexe, donc sur les personnages, ne suffit pas pour parler d'un film. Dans cet article, le cinéma restera l'horizon de notre réflexion et c'est en le maintenant comme perspective que « genre » et « psychanalyse » seront abordés. Il s'agira de voir si et comment les études de genre peuvent renouveler le champ des études filmiques comme la psychanalyse l'a fait en mettant en partage sa boite à outils conceptuels. Nous nous appuierons pour cela sur le film d'Alice Guy Blaché, Algie The minor, produit par la Solax-film en 1912, qui raconte l'histoire d'un jeune homme dont l'identité de genre trouble l'ordre social.

Ce film pose de façon subtile la question de la différence. En une dizaine de minutes, il raconte l'histoire d'un jeune citadin, Algernon Allmore, interprété par l'acteur comique Billy Quirk (18731926), contraint par le père de sa fiancée de prouver sa virilité. Le défi consiste à partir à la conquête de l'ouest afin que lui soit accordée la main de sa bien-aimée. Le film suit la trame narrative traditionnelle des récits de parcours initiatiques (mise en place d'un défi à affronter, parcours d'épreuves à franchir en milieu inconnu et retour du personnage) et travaille la question de la différence aux niveaux des rapports sociaux de sexes, de la construction du récit et de la mise-enscène.

Insister sur la différence des sexes pourrait renvoyer exclusivement à la différence entre femmes et hommes. Or, la variabilité des figures de la différence ne peut être réduite à l'idée d'une différence « première » entre les hommes et les femmes. Cette notion de différence(s) sexuelle(s) enferme la pensée dans le cadre d'une opposition binaire (« la» femme comme différence de l'homme) qui rend impossible tout autre type de différences. Traiter exclusivement de la différence des sexes dénie celles qui existent entre les individus de même sexe, mais aussi celles qui animent un même sujet. Or, le cinéma invente des modes de figuration de la différence qui permettent de sortir de cette logique. S'il véhicule ou fabrique parfois des stéréotypes, il n'a pas toujours ramené les femmes et les hommes à une féminité ou à une masculinité discursive. Un personnage de cinéma n'est pas construit uniquement en tant que sujet sexuel : sa construction dans le genre se fait aussi à travers le langage et les représentations culturelles. Le cinéma sait que le genre n'est pas la propriété des corps mais qu'il est « un ensemble d'effets produits dans les corps, les comportements et les relations sociales $\gg{ }^{4}$. À partir de ce postulat, il devrait être possible de trouver une articulation entre genre, psychanalyse et cinéma.

La première scène du film d'Alice Guy Blaché présente les enjeux du récit. Dès le premier plan, la

4 Michel Foucault, La Volonté de savoir, Gallimard, Paris, 1976, p.119. « Le genre, comme la sexualité, n'est pas la propriété des corps ou quelque chose qui existe originellement chez les humains, mais (...) il est 'un ensemble d'effets produits dans les corps, les comportements et les relations sociales', pour reprendre Foucault, et ce grâce au déploiement d' 'une technologie politique complexe' » écrit Teresa de Lauretis (Théorie queer et culture populaire, éd. La dispute, 2007, p. 41). 
famille de la jeune femme fait corps contre Algie. Le père et un autre homme le narguent alors qu'il revendique la main de la jeune fille. Le père s'assoit à son bureau et rédige une lettre. L'ensemble des protagonistes se groupe autour de lui pendant qu'Algie attend. Le père se lève et lui tend la lettre que les spectateurs découvrent en insert : «Si Algie Allmore prouve qu'il est un homme, d'ici à une année, il pourra prendre ma fille pour épouse ». Le défi est ainsi lancé. Algie serre la lettre contre ses lèvres et sort du champ. Le père et son complice éclatent de rire dès qu'il a disparu. Le personnage principal est donc défini comme hétérosexuel mais ses manières en font la risée de l'assemblée familiale. Dans les plans qui suivent, Algie, qui se prépare à partir, apparaît comme un personnage dont le genre fait question au niveau vestimentaire, par sa gestuelle et par les objets qui lui sont attribués. En effet, il porte une veste à carreaux cintrée, une chemise à col arrondi et un chapeau à large bord, relevé au niveau du front tel une capeline. Il transporte sa petite valise du bout des doigts et y jette négligemment un minuscule mouchoir à dentelle avant de glisser un revolver miniature à sa ceinture. Par ailleurs, aussitôt descendu du train qui l'a conduit dans l'ouest, Algie embrasse sur la bouche les deux cowboys à qui il demande son chemin. Algie serait la première figure de sissy, c'est-à-dire d'homme «efféminé »" , de l'histoire du cinéma. Il est effectivement différent des hommes et des femmes des milieux sociaux dans lesquels il évolue. Son costume et sa gestuelle le démarquent et en font un objet de regard et de moquerie. L'insistance sur les objets miniatures (mouchoir, valise et révolver) le renvoie à l'univers de l'enfance (celui des jouets) mais aussi à la dimension symbolique de la castration (la miniaturisation de l'arme renvoyant au risque de la réduction du pénis $\left.{ }^{6}\right)$. L'enjeu de la partie centrale du film sera donc de « rééduquer » Algie pour le réassigner au genre masculin.

L'éducation à la virilité d'Algie est confiée, dès son arrivée, à Jim, un grand moustachu alcoolique. Jim transmet à Algie le savoir minimal d'un chercheur d'or comme le maniement du revolver ou l'équitation. Mais en retour, Algie s'occupe de Jim quand ce dernier rentre ivre, ce qui n'était pas prévu dans le contrat éducatif. Une année s'écoule dans une camaraderie complice entre les deux hommes. Au moment de retourner dans l'est, Algie propose à Jim de l'accompagner et les deux hommes rendent au père de la jeune fille l'humiliation qu'il avait fait subir à Algie en entrant chez lui à coups de revolver. Le film ne remet donc pas en cause l'ordre social et la binarité des sexes. Si l'on s'en tient uniquement à la courbe narrative, Algie effectue le trajet qui lui permet d'entrer dans le monde social par le passage du mariage hétérosexuel. Cependant ce parcours est construit sur un travail narratif et formel qui fait de la différence une figure variable.

5 Roth-Bettoni note qu'avant la période de censure du code Hays (1934), «les sissies sont rarement représentés comme des personnages négatifs. Beaucoup moins en tout cas que la plupart des autres minorités telles qu'elles apparaissent dans le cinéma hollywoodien et qui subissent un sort caricatural » p.40 , L'Homosexualité au cinéma, éd. La Musardine, 2007

6 Sur la miniaturisation des objets, voir l'analyse que R.Bellour propose du minuscule rasoir utilisé par Hitchcock dans La mort aux trousses. "Le blocage symbolique" dans L'Analyse du film, éd. Calman-Lévy, 1995, p.147-149 
La construction narrative joue sur un récit symétrique qui fonctionne par effets de chiasme. Le film se compose d'une série de saynètes, intercalées par six cartons, qui se répondent symétriquement deux par deux. À la scène d'introduction que nous avons décrite plus haut répond la scène finale du retour d'Algie. Un carton annonce le retour du personnage : «De retour dans l'est » mais souligne aussi son changement de manières : «Sonner à la porte à la façon de l'ouest ». En effet, devant la porte de la maison, Jim tire un coup de feu. À l'intérieur, le majordome tombe à la renverse. Dans le salon, les protagonistes s'affolent. Jim donne un coup d'épaule dans la porte et les deux hommes entrent en force. Le majordome, sous la menace de l'arme, entre à reculons, introduisant malgré lui Jim et Algie qui retrouve sa fiancée. Algie harangue alors le père et lui rend la lettre qui les liait. Ce dernier se détourne mais Jim qui le dépasse d'une tête le menace de son arme. Le père se tourne alors vers Algie qui fait également valoir son revolver. Algie est toujours Algie mais ne ressemble plus tout à fait au personnage initialement moqué. On retrouve le lieu et les personnages du début, cadrés à l'identique et dans la même situation, mais le rapport de force est inversé. La place d'Algie dans cette famille est donc bien une construction et non une donnée de nature, «traversées comme toute construction sociale par des rapports de domination et des résistances à cette domination $»^{7}$.

Le travail formel des jeux de différences met clairement à jour ces rapports de force. Dans le film, deux autres scènes se répondent en symétrie : les scènes de saloon. Alors qu'Algie vient d'arriver dans l'ouest, il embrasse deux inconnus. En fait les deux hommes, sidérés par l'apparence et l'attitude d'Algie, décident de l'attraper par les bras, le soulèvent et l'emmènent dans le saloon où ils le déposent sur une table. Algie fait attraction. Sa différence suscite la curiosité, il est une créature, un freak que l'on peut déplacer comme un objet et donner en pâture aux autres pour se divertir. Tous les hommes se groupent autour de lui, l'observent et rient de son costume, de son chapeau, de son air. Algie commence par éternuer, s'essuie les sourcils et sort son mini-revolver. Un inconnu hilare, qui semble être un des meneurs du bar, s'en empare et tire dans le dos d'un homme sans que les balles n'aient plus d'effet qu'un chatouillement. Les autres rient et l'homme de dos s'approche. Il s'agit de Jim à qui Algie est confié. Contrairement aux scènes d'introduction et de conclusion, l'espace n'est pas ici celui du plan tableau, mais un espace conçu dans la profondeur de champ avec une ligne de fuite qui dessine les lignes de tension narrative. La table où est assis Algie se trouve à l'avant-gauche du cadre et la porte du saloon est cadrée dans le fond droit de l'image. Tous les trajets et déplacements sont organisés sur cette diagonale gauche-droite. Jim, dépité de la tâche qu'il va devoir accomplir, s'éloigne vers la porte qu'il ouvre laissant Algie passer devant lui, tandis que les autres hommes s'esclaffent de sa démarche. Jim leur tourne le dos et claque la porte.

Après cette scène de rencontre, dans la chambre qu'ils vont partager, Jim prête à Algie un « vrai »

7 G.Sellier et N.Burch, Le cinéma au prisme des rapports de sexe, Paris, éd Vrin, 2009 
revolver. À la vue de l'arme, Algie s'évanouit. La métaphore phallique est directe et l'évanouissement d'Algie, une réponse factuelle. Jim l'attrape et le jette sur son lit. Algie roule par terre. Démarre alors une période de compagnonnage. Un carton révèle que du temps a passé et qu'Algie commence à remplir son contrat. Dans leur chambre, alors qu'Algie nettoie le revolver que Jim lui a donné, Jim est ramené ivre par deux hommes. Algie désormais habillé comme les autres, voit son compagnon en plein délire souffrir d'hallucinations. Cette fois, c'est Algie qui couche Jim.

Dans la deuxième scène du saloon, Algie intervient pour empêcher Jim de boire. Alors que les autres hommes sont attablés, Jim et Algie entrent dans le bar. Jim s'approche d'eux. Ils lui tendent un verre. Jim refuse. Les hommes insistent. Jim tend la main, prêt à accepter, mais Algie s'interpose violemment. Il force l'homme qui tentait Jim à s'asseoir, prend Jim par la manche et l'entraine hors du saloon sans quitter des yeux ses ennemis. Ici aussi la mise en scène se déploie dans la profondeur de champ, ce qui renforce l'effet de chiasme sémantique. Aux manières de sissy d'Algie répond la tendance alcoolique de Jim, chacun venant atténuer le rapport problématique de l'autre au registre de la virilité. Alors qu'Algie était confié à Jim lors de son arrivée, c'est désormais lui qui veille sur son ami. Algie s'éloignait en se dandinant sous les rires sarcastiques et Jim s'éloigne penaud dans le silence. La mise en scène du spectacle de la différence et celle de l'abus de faiblesse est désamorcée par l'usage de l'espace, les entrées et sorties de scènes qui permettent aux personnages de s'associer pour inventer leurs propres solutions et résister aux humiliations des rapports de force.

Un plan du film est fondamental dans ce parcours. Algie n'est pas l'objet d'une révolution intérieure qui modifierait littéralement son attitude. Comme le dit un des cartons du film, il « remplit son contrat », mais il le fait au contact de Jim et à condition de ramener son complice avec lui dans l'est. Un plan, filmé en extérieur, puise avec inventivité dans les possibilités de la mise en scène des entrées et sorties de cadre. Il s'agit d'un plan autonome qui décrit Algie et Jim dans leur activité de chercheurs d'or. Armés de pioches, ils extraient des minerais de la terre. Alors qu'ils se retournent pour examiner leur collecte, la caméra recule et effectue un léger panoramique vers le haut qui décrit l'arrivée de deux bandits sur la colline. En bas du cadre, Jim et Algie se réjouissent de leur découverte pendant que les deux inconnus manigancent en haut de l'image. Jim met le précieux caillou dans sa poche et envoie Algie chercher un sac, mais il prend soin de lui confier son arme. Algie sort du cadre par la droite pendant que Jim se remet à creuser. La caméra pivote légèrement à droite pour suivre les bandits qui s'approchent de Jim à son insu. Les deux hommes se jettent sur lui et le neutralisent malgré sa forte carrure. Algie bondit dans le cadre et les menace à l'aide des deux revolvers. Les bandits sortent chacun d'un côté de l'image. Cette scène tournée en un seul plan est la seule où les deux personnages sont traités à égalité : pas de rapport de force entre eux, aucun n'est l'objet d'une soumission forcée, chacun fait sa part, mais aucun ne s'en sortirait sans l'autre. 
L'histoire du cinéma a produit des récits et des formes filmiques dans lesquels des schémas narratifs et les fonctionnements formels, basés notamment sur la loi du désir, la différence des sexes, la femme comme spectacle répondent à la psychanalyse. Mais un court-métrage comme celui-ci montre que le cinéma fait également partie des lieux qui « reconfigurent inlassablement, (...) les normes sexuées qui favorisent et légitiment la domination masculine $»^{8}$. La manière dont Algie fait circuler les marqueurs de genre et la manière dont le film pense formellement la question de la différence, rendent les positions instables et inassignables et déconstruit les normes sexuées. Ces normes ont fait de lui la risée de la société, mais sa droiture et sa force morale en font finalement un être riche et sensible. Son rire lorsque Jim tire des coups de feu chez ses beaux-parents déconstruit l'usage viriliste des armes. Les coups de feu le font rire car il sait qu'ils sont tirés non pour tuer, mais pour renverser une construction sociale qui lui refusait une place digne. Son voyage dans l'ouest lui aura appris que cette construction n'était qu'une mascarade. Le film répond ainsi à la question que pose Judith Butler en 1990 : « dans quelle mesure le corps vient-il à exister grâce au(x) marque(s) du genre ? $»^{9}$ en rappelant la façon dont les corps sont niés quand les marques de genre qu'ils ont choisi excèdent la place à laquelle ils ont été assignés. Le cinéma des années 1910-1920 a expérimenté la performativité des personnages filmiques et développé sa capacité à inventer de nouveaux sujets. Mais en 1930, pour les rédacteurs du code Hays (appliqué de 1934 à 1966), il faut briser cette inventivité subversive. Laisser de côté les études de genre dans la réflexion sur l'esthétique du cinéma nous priverait de penser ce lien entre construction du sujet et inventivité filmique.

8 N.Burch et G.Sellier (2009), p.13

9 J.Butler, Trouble dans le genre (1990), éd la Découverte 2005, p.72 